\title{
Characterization, modeling and simulation of the MIMO propagation channel
}

\author{
Ghaïs El Zein ${ }^{\mathrm{a}, *}$, Hanna Farhat ${ }^{\mathrm{a}}$, Patrice Pajusco ${ }^{\mathrm{b}}$, Jean-Marc Conrat ${ }^{\mathrm{c}}$, \\ Yves Lostanlen ${ }^{\mathrm{d}}$, Rodolphe Vauzelle ${ }^{\mathrm{e}}$, Yannis Pousset ${ }^{\mathrm{e}}$ \\ a Institut d'Electronique et de Télécommunications de Rennes, IETR-UMR CNRS 6164, INSA, 35043 Rennes cedex, France \\ ${ }^{\mathrm{b}}$ Télécom Bretagne, UMR CNRS 3192 Lab-STICC, Technopôle Brest Iroise - CS 83818, 29238 Brest cedex, France \\ ${ }^{\mathrm{c}}$ Orange Labs, 6, avenue des Usines, BP 382, 90007 Belfort Cedex, France \\ ${ }^{\mathrm{d}}$ SIRADEL, 1920 Yonge Street, $2^{\text {nd }}$ floor, Toronto, ON M4S 3E2, Canada \\ ${ }^{e}$ University of Poitiers, XLIM-SIC Laboratory, UMR CNRS 6172, 86962 Futuroscope Chasseneuil Cedex, France
}

\begin{abstract}
This paper deals with several aspects relative to the MIMO propagation channel. Based on simulations and/or measurements, different approaches are used to model the propagation channel. These models are useful for the MIMO system design. Several studies are performed in order to realize realistic simulation of MIMO channel. Different measurement techniques are used in characterizing the propagation channel in various environments. Measurement campaigns made in different situations have been analyzed to obtain the relevant statistical parameters of the channel. Simulation of MIMO channel is then presented. Measurement and simulation results provide an evaluation of the capacity of MIMO channel. Obtained results show feasibility in the integration of MIMO techniques in practical wireless communication systems.
\end{abstract}

\section{Résumé}

Caractérisation, modélisation et simulation du canal de propagation MIMO. Ce papier traite de plusieurs aspects relatifs au canal de propagation MIMO. Différentes approches, basées sur des simulations et des mesures, utilisées pour modéliser le canal sont d'abord présentées. Ensuite, les différentes techniques de mesure utilisées dans le but de caractériser le canal de propagation dans divers milieux sont décrites. Des campagnes de mesures effectuées dans différents environnements sont analysées pour obtenir les paramètres statistiques du canal. Quelques problématiques liées à la simulation du canal MIMO sont évoquées notamment en lien avec une simulation réaliste dans des milieux complexes. Les résultats obtenus, en simulation comme en mesure, permettent une évaluation de la capacité du canal MIMO. Ces résultats permettent de discuter de l'intégration des techniques MIMO dans des systèmes de communication sans fil.

Keywords: Propagation channel; Characterization; Modeling; Simulation; Capacity; MIMO

Mots-clés : Canal de propagation ; Caractérisation ; Modélisation ; Simulation ; Capacité ; MIMO

\section{Introduction}

In the last decades, the world of telecommunications has known an important development, driven by the success of $2 \mathrm{G}$ mobile communications systems such as GSM and by the global Internet phenomenon. The transition to $3 \mathrm{G}$ has relied on a migration path that defined a way to integrate multiple services such as multimedia, packet switching and wideband radio access. In this context, Multiple-Input Multiple-Output (MIMO) technology is recognized as a good solution in the development of the forthcoming generation of broadband wireless networks. This technique is essential for digital communications to increase data rates and/or to improve system performance [1].

The purpose of this article is to highlight various aspects concerning the characterization and the modeling of the propagation channel which are particularly critical in MIMO system design. At first, Section 2 presents a brief overview of the wireless communication systems and introduces the principles of MIMO systems. In Section 3, the different approaches used to model the radio propagation channel are synthesized. Section 4 describes a measurement system, used to characterize the MIMO propagation channel, and presents some experimental results. Section 5 addresses MIMO channel simulation, which leads to an assessment of the channel capacity. In Section 6, results of measurements are presented and analyzed for a possible integration of MIMO techniques in future wireless systems. Section 7 concludes this paper.

\footnotetext{
* Corresponding author.

E-mail address: ghais.el-zein@insa-rennes.fr (G. E1 Zein)
} 


\section{MiMo wireless communication systems}

Nowadays, wireless access networks show limits in terms of data rate, quality of service (QoS), and spectral efficiency. Many applications are concerned such as wireless local area networks (WLAN), broadband wireless access (BWA), and future generations of mobile radio systems (3G, LTE-A and beyond). For several years, efforts have been made to improve the design of the existing systems. The trend to increase data rates will most probably continue to reach 1 Gbps considering a moderate mobility. The MIMO technology appears as a new concept to fulfill those specifications. Since time and frequency domains processing are pushed to their limits, the space domain can be exploited. The MIMO principle can be simply defined as transmitting multiple streams of data on multiple antennas at the same frequency. The use of multiple receiving antennas improves the system performance.

Ideally, the channel capacity linearly grows with the number of transmitting (Tx) and receiving (Rx) antennas [1]. This technique can be viewed as a generalization of space diversity and smart antennas [2]. It supposes a channel rich in multiple paths, in order to exploit independent transmission channels between the Tx and Rx antenna arrays. This transmitting and receiving structure can be modeled using a matrix representation of the channel.

Information theory shows that two fundamental mechanisms are at stake in the process of transferring data:

- Diversity which is based on the reception of multiple decorrelated copies of the same transmitted information.

- Multiplexing which is based on the reception of multiple independent symbols of information.

These two mechanisms, diversity and multiplexing, combat fading effects and increase the channel capacity respectively [3]. The large improvement in link reliability and/or data rates, predicted by information theory, relies on a fine knowledge of the propagation phenomena. Such knowledge makes it possible to choose the most appropriate coding/modulation scheme for a given environment. Transmitting and receiving antenna arrays have to be carefully designed to maximize the channel rank; i.e. the number of eigenmodes available for communication. In this case, correlation and dispersion measurements of channel parameters play a central role.

\section{MiMo channel modeling}

The radio propagation of electromagnetic waves from a transmitter to a receiver is characterized by the presence of multipath due to various phenomena such as reflection, refraction, scattering and diffraction. The performance of MIMO systems is largely dependent on the propagation medium and on the structure of the antenna array. In this context, both the space-time characterization and modeling of the channel are essential. Thus, one objective of our studies aims to bring a better understanding of the MIMO propagation channel leading to a realistic channel model. Several methods of classification of the models are proposed in the literature [4-9]. In this paper, we distinguish between deterministic and stochastic models.

\subsection{Deterministic models}

Deterministic models are based on a fine description of a specific environment. Two main approaches can be identified:

- $\quad$ Ray-tracing models, which are based on optical approximations, need complete geometrical and electromagnetic specifications of the simulated environment. They enable to estimate the channel characteristics with a good accuracy, if the environment modeled is not too complex. This method is generally based on a 3D description of the environment. A simplified modeling allows reducing significantly the simulation time and computational resources. A double directional description of the radio channel [10] results from this kind of simulator. Moreover, other models can be used which are based on the Maxwell's equations; they require much more computation time.

- $\quad$ Recorded measurement data can be played back by means of computer. Thus, the measurement campaigns of the propagation channel enable to extract different characteristic parameters of a specific environment. But these parameters are specific to experimental conditions including the environment and the antenna array. Furthermore, the simulations need large memory resources.

\subsection{Stochastic models}

The stochastic models describe the channel parameters by random laws. Many possible implementations of stochastic MIMO models can be found like the geometrically-based and correlation-based models. The geometrical models consider a statistical distribution of scatterers around both, the base station and the mobile. Assuming a single bounce or double bounce, the channel model can be computed. These models also allow channel change versus time by computing the movement of the transmitter or the receiver.

The COST 259 Directional Channel Model [10-12], and its successors the COST 273 [13-15] and COST 2100 [16] offer examples of statistical MIMO channel models. Recently, other models like WINNER II, taking into account new environments, were also proposed in the literature [17,18]. 
The most commonly used models in communication chain simulations are certainly the Gaussian stochastic models that are based on correlation between elements of the channel matrix. The complete model consists of determining the correlation matrix between all the channels. This adds complexity in terms of physical interpretation.

For this reason and to facilitate the extension of existing SIMO (Single-Input Multiple-Output) and MISO (MultipleInput Single-Output) models, other models separating the correlations at Tx and Rx have been used. In these models, a separable covariance structure (referred as the Kronecker product model [19]) can be created with the Kronecker product of the covariance matrices for signals on the transmitting and receiving arrays. Published results have demonstrated key deficiencies in these models [20]. Another model, developed and analyzed in [21], assumes a certain relationship between the second order properties in Tx and Rx. This model, based on the definition of structured vectorial modes and the definition of a coupling matrix, allows a greater flexibility in the degree of diversity and multiplexing offered by the channel.

Other studies have shown that a keyhole phenomenon was theoretically possible (e.g. in a corridor, ...), when the waves are propagated through the same hole. Measurement campaigns are also realized in such confined environments like tunnels [22].

All these published results show the importance of the research on MIMO channel modeling. The main objective is to obtain an accurate and realistic channel model.

\section{Mimo channel characterization}

The study of wave propagation appears as an important task when developing a wireless system. The analysis is usually conducted in the time domain, which allows measuring the coherence bandwidth, the coherence time, the respective delay spread, and Doppler spread values. Also, coherence distance and wave direction spread are used to highlight the link between propagation and system in the space domain. For broadband systems, the analysis of both path loss (estimation of cell coverage and carrier-to-interference ratio) and impulse response (estimation of the wideband channel characteristics) are required. Therefore, an accurate description of the spatial and temporal properties of the channel is necessary for the design of broadband systems, and also for the choice of the network topology.

To meet this goal, a theory was first proposed in [23], in the time domain, and then extended to a spatio-temporal characterization in $[24,25]$. This theory helps define the relationship between the different possible representations of the propagation channel. Therefore, from these representations, we can extract specific information related to the degree of spatio-temporal coherence or dispersion of the channel. However, these parameters characterizing the channel are often defined by assuming the Wide-Sense Stationary and Uncorrelated Scattering (WSSUS) hypothesis, which implies that the spatio-temporal variation of the average power of the signal on a small scale can be neglected. Considering the MIMO transmission channel, defined by the propagation channel (medium) and the antenna arrays at Tx and Rx, several parameters can be exploited as the eigenvalues of the channel correlation matrix, and the channel capacity.

\subsection{MIMO channel characteristics}

\section{- $\quad$ Singular value decomposition (SVD)}

The classical channel representation considers $N_{R} \mathrm{X} N_{T}$ SISO (Single-Input Single-Output) channels (Fig. 1) and is modeled by the channel matrix $\mathbf{H}$. The diagonalization of the matrix corresponds to expressing the channel as the superposition of several parallel and uncorrelated sub-channels (or eigenmodes) where each channel carries a fraction of the transmitted signal. Thus, the diagonalization is carried by the singular value decomposition of the matrix $\mathbf{H}$, as shown by Eq. (1):

$$
\mathbf{H}=\mathbf{U} \Sigma \mathbf{V}^{H}
$$

The diagonal matrix $\Sigma\left(N_{R} \times N_{T}\right)$ contains $N$ real positive or null singular values $\sigma_{k}$ of the channel matrix $\mathbf{H}$, which are related to the eigenvalues $\lambda_{k}$ of the correlation matrix $\mathbf{H} . \mathbf{H}^{H}$, by $\sigma_{k}{ }_{k}=\lambda_{k}$. $\mathbf{U}$ and $\mathbf{V}$ are unitary matrices and (.) ${ }^{H}$ means transpose-conjugate. As shown in Fig. 1, the singular value decomposition of the channel allows us to assimilate the more or less correlated $N_{R} \mathrm{x} N_{T}$ propagation channels, to $N\left(N=\min \left(N_{R}, N_{T}\right)\right)$ completely uncorrelated channels. The singular values tend to zero when the correlation increases. As long as the decomposition provides an equivalent representation of the channel $\mathbf{H}$, the transfer coefficients $\lambda_{k}$ retain the same total power given by Eq. (2):

$$
\sum_{k=1}^{P} \lambda_{k}=\|\mathbf{H}\|^{2}=\sum_{i=1}^{N_{R}} \sum_{j=1}^{N_{T}}\left|\widetilde{H}_{i j}\right|^{2}
$$

This decomposition shows that at most $P$ antennas receive a signal. Other antennas (from $P+1$ to $N$ ) associated with null singular values only receive noise. In this equivalent representation, the actual number of antennas receiving a signal is limited to $P$, the rank of the matrix $\mathbf{H}$. A key element associated with the performance of a MIMO system is the spacing between antennas. Indeed, especially for small size systems, antennas in close proximity present the risk of having strongly correlated signals. 


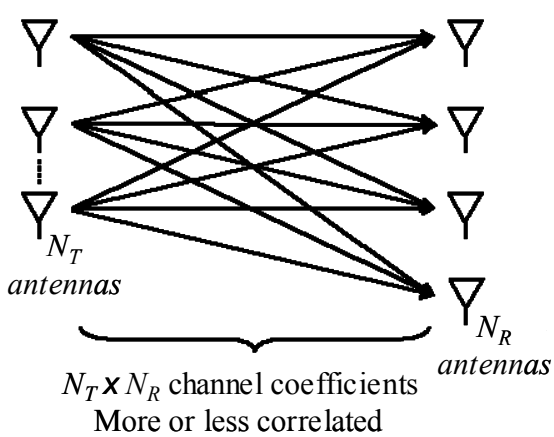

(a)

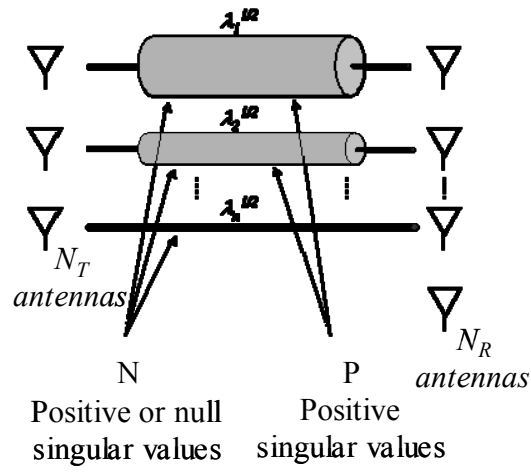

(b)

Fig. 1. Decomposition of a MIMO channel into independent sub-channels by decomposition in singular values.

(a) Classical correlated channels; (b) Independent sub-channels representation.

\section{- MIMO channel capacity}

Ergodic capacity is the second important characteristic parameter. It evaluates the performance of MIMO channels by quantifying the maximum information rate able to be transmitted through the propagation channel without error. Assuming equal power on each transmitting antenna, Eqs. (3) and (4) define this parameter, in bit/s/Hz:

$$
\begin{aligned}
C_{\text {MIMO }} & =E\left[\log _{2}\left[\operatorname{det}\left(\mathbf{I}_{N_{R}}+\frac{\rho}{N_{T}} \mathbf{H} \cdot \mathbf{H}^{H}\right)\right]\right] \text { if } N_{R} \leq N_{T} \\
C_{\text {MIMO }} & =E\left[\sum_{i=1}^{P} \log _{2}\left(1+\frac{\rho}{N_{T}} \lambda_{i}\right)\right]
\end{aligned}
$$

where $\rho$ is the Signal to Noise Ratio (SNR), $\lambda_{i}$ are the eigenvalues of $\mathbf{H} . \mathbf{H}^{H}, \mathbf{I}_{N_{R}}$ is an $N_{R} \mathrm{X} N_{R}$ identity matrix, and $\mathrm{E}\{$.$\} is the expected value.$

\subsection{Measurement system and results}

In practice, two main approaches can be used in order to characterize the propagation channel [26]. The first approach directly measures the time-variant MIMO channel coefficients matrix simultaneously in the time, frequency, and spatial domains. However, this method presents some limitations as the antenna arrays used during measurement must be assumed for system simulations. The second approach, based on the processing of the different time-variant transfer function and generally referred to as double directional channel measurements [27], tends to estimate the multipath parameters (direction of departure: DoD, direction of arrival: DoA, time delay, Doppler shift, polarization and amplitude) by using high resolution multidimensional MIMO sounding techniques. With the polarization parameters knowledge, this approach excludes the antenna and electronic influences from the measured results and thus, it can be easily generalized. Nevertheless, the choice of antenna array architecture remains very important to accurately resolve the multiple paths in azimuth, elevation, and polarization.

\section{- Measurement system}

For our measurements, a wideband channel sounder developed in the IETR laboratory was used (Fig. 2) to characterize the double directional channel [28]. It operates at $2.2 \mathrm{GHz}$ (close to $3 \mathrm{G}$ and WLAN bands) and $3.5 \mathrm{GHz}$ (WiMAX). It uses a periodic transmit signal based on the direct sequence spread spectrum technique. This dual-band sounder offers a temporal resolution of 11.9 ns with a $50 \mathrm{~dB}$ dynamic range on the impulse response, $90 \mathrm{~dB}$ power range with an Automatic Gain Control (AGC), and a bandwidth of $100 \mathrm{MHz}$. In order to estimate the propagation parameters of channel multipath components, various high resolution algorithms (Unitary ESPRIT, SAGE) are used.

For outdoor measurements at $2.2 \mathrm{GHz}$, we used 4 and 8 active elements ULA (Uniform Linear Array) (Fig. 3(a)) respectively at Tx and Rx. A 16 active elements URA (Uniform Rectangular Array) has also been developed (Fig. 3(b)). This antenna array enables the characterization in azimuth and elevation plans in order to be used for indoor and penetration configurations. For measurements at $3.5 \mathrm{GHz}$, UCA (Uniform Circular Arrays) have been developed. Figs 3(c) and 3(d) show the UCA Tx antenna with 4 elements and UCA Rx with 16 elements. These antenna arrays accelerate the collection of measurement; they can scan all directions in the horizontal plane, simultaneously.

The high-resolution algorithm Unitary ESPRIT [29] (Estimation of Signal Parameters via Rotational Invariance Techniques) is suitable for planar-type architectures. We have also chosen to develop the SAGE algorithm [30] (SpaceAlternating Generalized Expectation - Maximization). This algorithm is more suitable when using circular arrays [31]. 


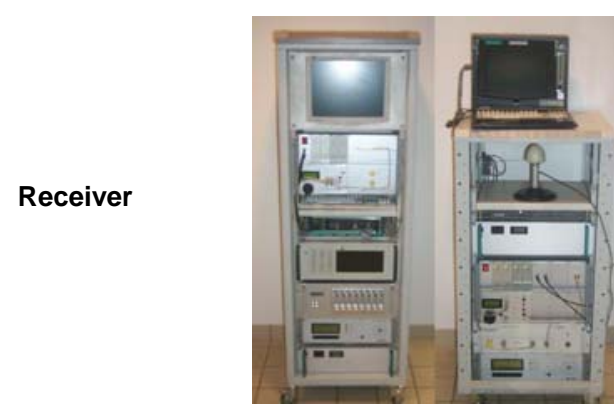

\section{Transmitter}

Fig. 2. MIMO channel sounder.

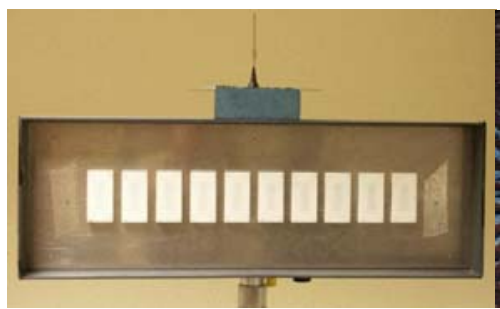

(a)

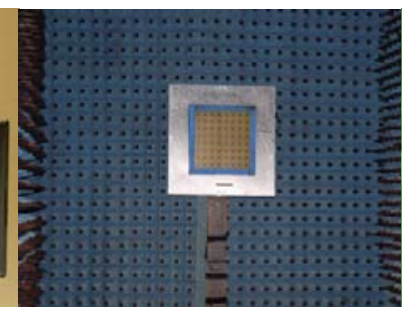

(b)

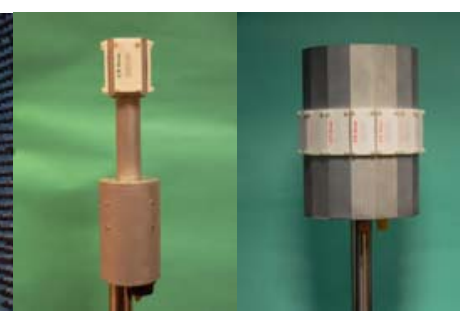

(c)

(d)

Fig. 3. (a) ULA Rx antenna (8); (b) URA Rx antenna (4 × 4); (c) UCA Tx (4); (d) UCA Rx (16).

\section{- Measurement results}

A measurement campaign in an Outdoor-to-Indoor environment was conducted at $3.5 \mathrm{GHz}$ [32]. The transmitter was placed on the rooftop of a building and the receiver at different positions in a second building. Fig. 4 presents the different Rx positions and the measured DoA results obtained with the SAGE algorithm. For each measurement point, a polar representation illustrates the received power (proportional to the length of a segment). Based on the obtained results, one can notice that the spread in this type of environment, and in the case of non-visibility (NLOS : Non Line Of Sight) between the transmitter and receiver, marked privileged paths of propagation through the radio openings (windows and doors). Moreover, these measurements have highlighted the importance of diffraction on the vertical frames of the windows.
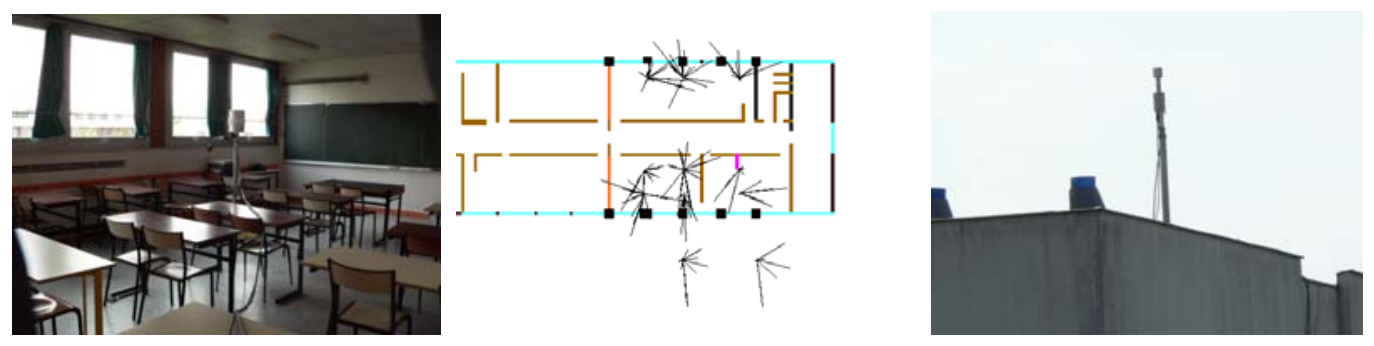

Fig. 4. Outdoor-to-indoor scenario and DoA results.

\section{Mimo channel simulation}

MIMO channel simulators are very important in the design of MIMO wireless systems. Using geographical data base, it is possible to predict space-time characteristics of the channel impulse response. Such simulators make it possible to compare propagation measurements and predictions. This enables to investigate new environments where measurements are not available.

\subsection{Software simulator at physical level}

A 3D radio channel simulator has been developed at the XLIM-SIC laboratory. It is based on one hand, on the Geometrical Optic and Uniform Theory of the Diffraction and, on the other hand, on optimized ray tracing techniques. For a given transmitter-receiver link, the various existing paths and their parameters (attenuation, delay, polarization, DoA, DoD ...) can be estimated. So, these simulations can provide the evolution of the channel impulse response (CIR) for a chosen series of reception points, in either indoor or outdoor environment.

This tool is coupled with another one dedicated to the channel characterization (as defined in $\S 4.1$ ). The combination of the two simulators is illustrated by the diagram in Fig. 5, where the input information is relative to the radio link characteristics in the studied environment, and the output provides a complete SISO or MIMO channel characterization. Note that the CIR, DoD, and DoA were extracted from the simulation and used in the characterization procedure [33-35]. 


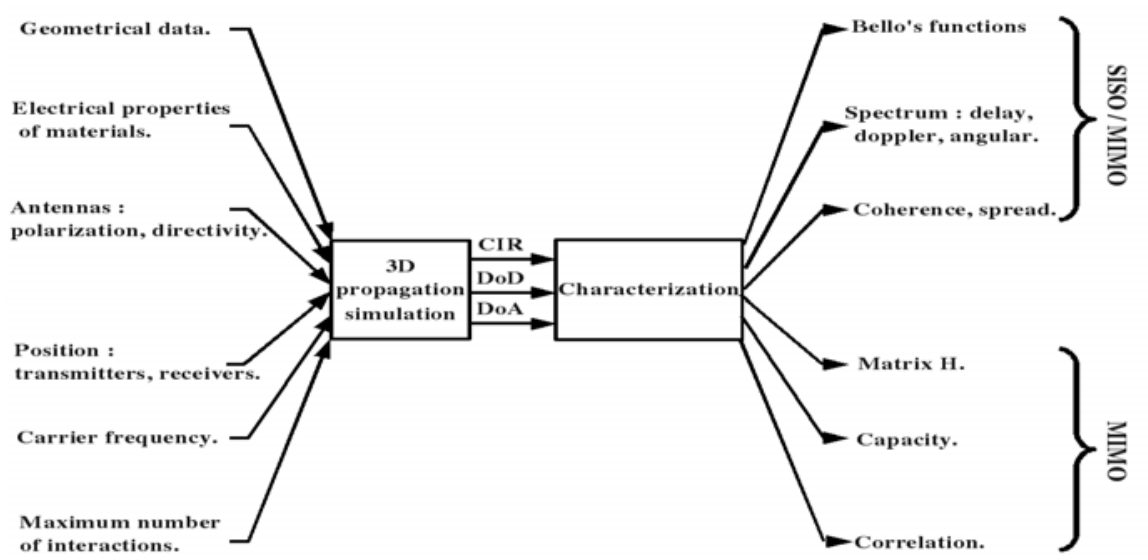

Fig. 5. Association between MIMO simulation and channel characterization.

To define correctly some input parameters in order to optimize the channel modeling, it is possible to study, for example, the influence of some electromagnetic interactions (Fig. 6), like reflection (R), diffraction (D), and transmission $(\mathrm{T})$ on the simulated power delay profile. For this study, measurements are considered as the reference.

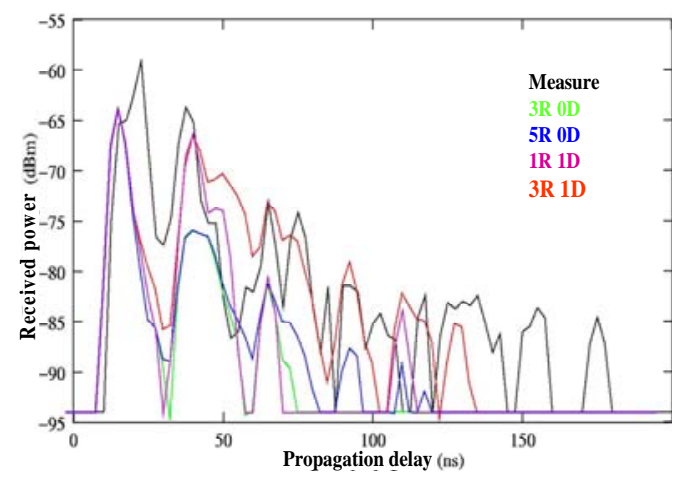

Fig. 6. Example of electromagnetic interactions influence on a power delay profile in an indoor configuration.

Concerning the influence of the environment description on the channel characterization, a small scale study and a large scale study can be realized. The first one analyzes the impact of an accurate description of some realistic walls on the characterization of SISO and MIMO channels. The second one addresses this issue by studying the influence of the description of indoor realistic environments.

In the first approach, the investigation focuses on the geometry, electrical, and periodicity of walls structures. It is shown that such structures consider adding constructive and destructive interference in the received signal, which can affect the quality of communications. Thus, for a MIMO channel, the statistical survey conducted in [36,37] allows to analyze the effect of these heterogeneous walls on the modeling of the channel at $900 \mathrm{MHz}$. A notable difference is shown on the received power distribution and on the channel capacity for heterogeneous walls and homogeneous walls, whatever the number of transmitter and receiver antennas.

In complex indoor environment, this type of small scale study is almost impossible or very time-intensive. So, the main objective of the second approach is to evaluate the degradation of the simulations accuracy according to the electrical and geometrical description of the environments [35-37]. Two indoor environments have been considered (Fig. 7) and four levels of description are defined.

Level (A) is the simplest and consists of two rectangular parallelepipeds with concrete walls, including transmitting and receiving antennas. Level (B) considers the previous volume of level (A) with geometric descriptions consistent with the architectural plan (taking account of doors, windows, etc.) and electrical properties of the elements taken similar to the concrete ones. Level (C) takes into account all the rooms lying within or not the transmission area. These are geometrically well described but their electrical properties are similar to those of the concrete. Finally, level (D) corresponds to the highest detailed description of both geometry and electrical.

The impact of the environment description accuracy on the channel characterization has been shown on the capacity gain. This gain results from the normalization of the average capacities relative to the SISO transmissions. Moreover, these equivalent SISO capacities are obtained for the highest level of description (level D). The antennas spacing and number have been considered (without mutual coupling between antennas) to define several MIMO configurations. It is observed in Fig. 8 that a low level of description (A) introduces significant errors in the simulations. However, it is not necessary to consider a high level. The level (B) constitutes a trade-off between the computation time and the accuracy of the channel characterization. In this study, the authors also analyze the impact of polarization diversity on the performance of the channel. 


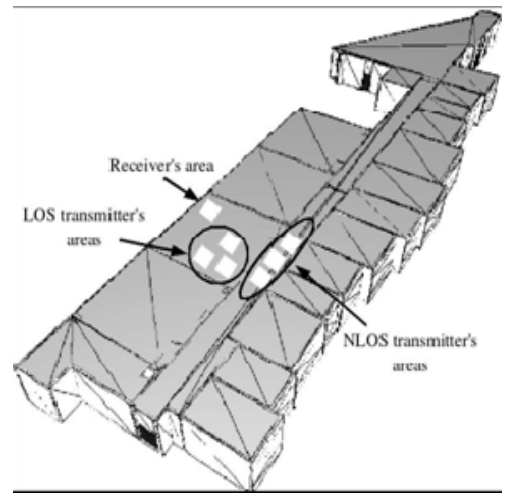

(a)

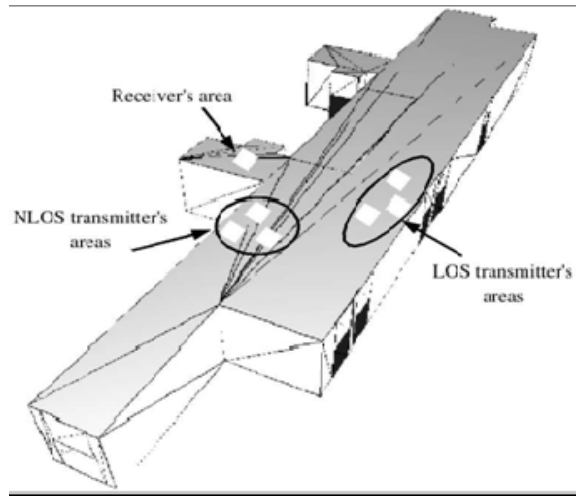

(b)

Fig. 7. Indoor propagation environments: (a) Stage of a building; (b) Large hall of a building.

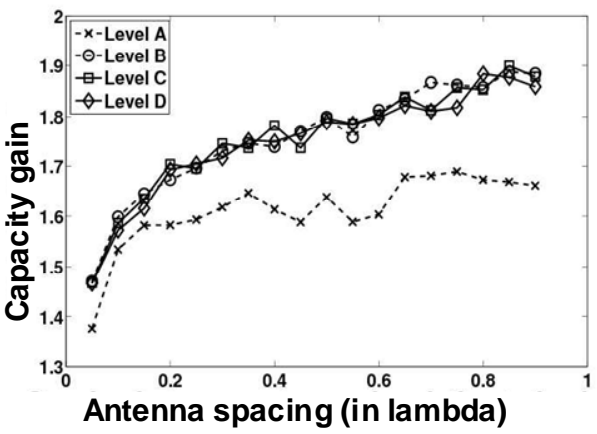

(a)

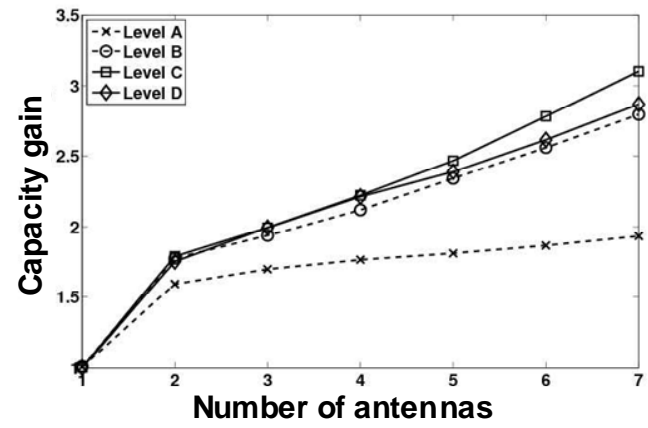

(b)

Fig. 8. Evolution of the capacity gain: (a) Effect of the antenna spacing; (b) Effect of the number of antennas.

\subsection{Propagation channel for link level simulations}

The previous simulator is dedicated to radio channel prediction. In link level simulations, more general models are required such as tapped delay line models or stochastic channel models. The challenge is to simulate, as fast as possible, any kind of model with any kind of antenna array configuration. To reach that aim, a versatile and efficient channel simulation software was developed. More details about this tool called MASCARAA are available in [38].

\section{- The ray concept}

The most generic approach to represent a MIMO propagation channel is to use the double directional radio channel concept introduced in [27]. The principle consists of representing the channel by a set of rays. The electromagnetic properties of a ray make it possible to determine the received field as a function of the transmitted field. By assuming the plane wave propagation hypothesis, the transmitted or received field is always perpendicular to the propagation direction. Thus, it is more convenient to express this field in the spherical base. Any type of polarization can be expressed with only $E_{\theta}$ and $E_{\phi}$ components. As the propagation channel causes different complex attenuation on $E_{\theta}$ and $E_{\phi}$ components, the received field at a specific frequency can be expressed by the matrix given by Eq. (5):

$$
\left(\begin{array}{l}
E_{\theta}^{R x} \\
E_{\phi}^{R x}
\end{array}\right)=\mathrm{A} \cdot\left(\begin{array}{l}
E_{\theta}^{T x} \\
E_{\phi}^{T x}
\end{array}\right)=\left(\begin{array}{ll}
a_{\theta \theta} & a_{\phi \theta} \\
a_{\theta \phi} & a_{\phi \phi}
\end{array}\right)\left(\begin{array}{c}
E_{\theta}^{T x} \\
E_{\phi}^{T x}
\end{array}\right)
$$

where $a_{\theta \theta}, a_{\theta \phi}, a_{\phi \theta}$ and $a_{\phi \phi}$ are four complex gain values that characterize the electromagnetic properties of the ray. To simplify the computation of the channel impulse response, the antenna gain and the ray gain are assumed to be constant over the whole simulation bandwidth. According to this common hypothesis, the channel impulse response for a SISO configuration is given by Eq. (6):

$$
h(t)=\sum_{k=1}^{N_{\text {Rays }}}\left(G_{\theta}^{M o b}(k) \quad G_{\phi}^{M o b}(k)\right) A(k)\left(\begin{array}{l}
G_{\theta}^{B t s}(k) \\
G_{\phi}^{B t s}(k)
\end{array}\right) \delta\left(t-\tau_{k}\right)
$$

where $N_{\text {Rays }}$ is the ray number, $G_{\theta}^{M o b}(k)$ and $G_{\phi}^{M o b}(k)$ are respectively the $E_{\theta}$ and $E_{\phi}$ components of the MS antenna gain in the direction of the $k^{\text {th }}$ ray, $G_{\theta}^{B t s}(k)$ and $G_{\phi}^{B t s}(k)$ are respectively the $E_{\theta}$ and $E_{\phi}$ components of the BS antenna 
gain in the direction of the $k^{\text {th }}$ ray. For usual wireless communication systems, the distance between antennas is much smaller than the distance between transmission devices and scatterers. A reasonable approximation is to consider that every SISO channel of a MIMO link has the same physical properties [39]. Eq. (6) can be easily extended to MIMO channels taking into account other antenna characteristics.

The above equations were dedicated for the static propagation channels. Channel variability can be obtained by generating a set of rays for each mobile location. This solution would obviously be very time-intensive. Considering the WSSUS hypothesis, the most efficient solution considers that ray characteristics remain unchanged, except for the phase, which is updated according to the mobile location. This solution is very similar to that adopted for the extension of SISO models to MIMO applications. The different locations of the mobile can be viewed as a virtual array.

Many propagation channel models for link level simulations are available: tapped delay line, ray tracing/launching, scattering or geometrical models... The basic idea of our approach is to represent a channel model by a set of rays. For instance, a tapped delay line model profile is described by a limited number of paths. Each path can be split into a subgroup of rays with a delay equal to the path delay. The cumulative power of sub-rays coming from the same path is equal to the path power. The common Doppler spectra are the Rayleigh spectrum (also designed by "classic"), the flat spectrum and the Rice spectrum [40,41]. A "classic" Doppler spectrum corresponds to a sub-group of rays with equal power and uniformly distributed on a horizontal plane (Clarke's model). The method implemented to calculate the DOAs at MS from a Doppler spectrum is based on conclusions of previous studies [42-45].

\section{- Optimization}

Many trigonometric functions are used to compute the gains of each ray. Trigonometric operations are timeconsuming functions. It is therefore time-saving to replace these functions by look-up tables that contain pre-computed values of cosine and sine functions. Another refinement is to use only integer type for phase representation. This speeds up computation and provides a direct index in the look-up table. These different refinements divided by 3 the computation time of the channel impulse response.

To perform the convolution, the channel impulse response has to be discrete-time form. The main problem of the continuous-to-discrete conversion is due to the fact that the ray delays are not multiples of the sampling period $T_{s}$. A well-known method to sample the impulse response consists in approximating the ray delay to the nearest multiple of $T_{s}$ [46]. Although this mapping method is very simple, it significantly modifies the space-time characteristics of the original channel and consequently the system performance. To reconstruct and sample the channel impulse response accurately, Eq. (7) is used:

$$
h(n)=\sum_{k=1}^{N_{\text {Rays }}} a^{\prime}(k) g\left(n T_{s}-\tau(k)\right)
$$

where $g$ is the shaping filter, with a unitary frequency response in the useful bandwidth and $a^{\prime}(k)$ is the gain of the $k^{\text {th }}$ ray. To avoid time-intensive computation of $g$, the $g$ function is oversampled and stored in a look-up table. Then, the discrete channel is convolved with a continuous input signal. The convolution is computed with the Over-and-Add method (OA method) [47,48], which is more efficient than other methods, such as direct and tap methods.

\section{- Library overview}

This channel simulator is written in C Ansi, which is easily portable on various Operating Systems or simulation platforms. The library is licensed under the GNU General Public License. It was developed in the framework of different national projects related to hardware channel simulation and MIMO transmission systems. An overview of the different modules of the library is presented in Fig. 9.

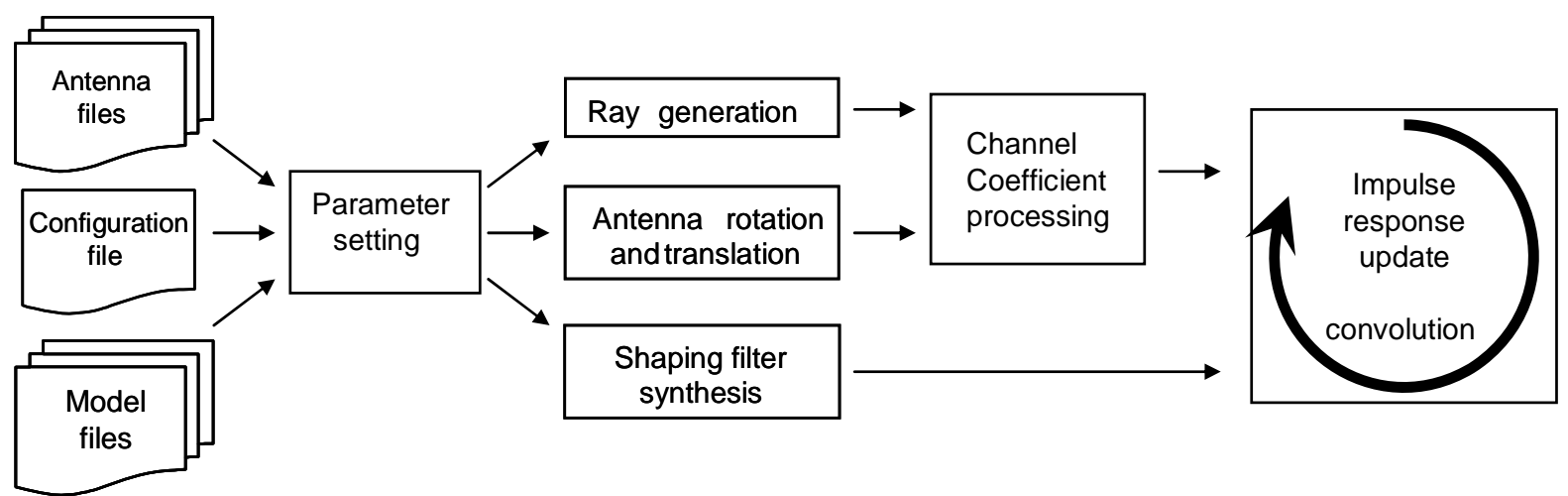

Fig. 9. Schematic of the channel simulator. 
To conclude, this library provides a versatile and efficient propagation channel simulator mainly dedicated to MIMO link-level simulations. The major characteristics of this library are recapped here:

o compliant with most propagation models (tapped models with arbitrary delays, scattering model, ray tracing, etc.)

o compliant with applicable standards (GSM, UMTS, WiFi, etc.)

o supports arbitrary geometry of antenna array (linear, circular, planar, etc.) with arbitrary polarization

o allows dynamic simulation

o optimization of time processing

o available on Windows, Linux, Unix

\section{Applications of MIMO technology}

The statistical analysis permits us to extract the second-order parameters of the propagation channel and to provide important characteristics for the system design, such as the coherence bandwidth, and the coherence distances at the transmission and reception sites. These various coherence parameters have a profound implication in the design of MIMO communication systems. In particular, antenna element numbers and inter-element spacing can be deduced from coherence distance. One can note that antenna spacing, within the transmitting and receiving arrays, must be larger than the local coherence distance to get sufficient transmit and receive decorrelation. Likewise, the transmit bandwidth must be larger than the channel coherence bandwidth to benefit from frequency diversity.

For example, a measurement campaign was carried at $2.2 \mathrm{GHz}$ (UMTS band) in a dense urban environment. The measurement results show that for a UMTS system with a $5 \mathrm{MHz}$ bandwidth, the channel offers little frequency paths diversity (less than $10 \%$ of the cases) [49]. Moreover, space diversity is obtained at the Rx base station by separating the antennas at distances close to $11 \lambda(1.5 \mathrm{~m}$ at $2.2 \mathrm{GHz})$. Also, the diversity at the Tx mobile position has been evaluated by spacing out the antennas at the distance $0.36 \lambda(4.9 \mathrm{~cm}$ at $2.2 \mathrm{GHz})$. It is therefore quite reasonable to consider the integration of spatial diversity at the mobile terminal.

\section{Conclusion}

In this article, we reviewed various aspects concerning the characterization and modeling of the MIMO propagation channel. Both deterministic and stochastic models have been presented. Concerning the channel characterization, the adopted method, based on double directional channel measurements, allows to resolve the channel parameters and to remove the antenna characteristics from the results.

Considering different practical situations, the extracted second-order statistical parameters of the propagation channel at $2.2 \mathrm{GHz}$ (UMTS) are used to highlight the connection between propagation and communication system in the space and time domains. Measurement results obtained at 3.5 GHz (WiMAX), for an outdoor-to-indoor scenario, show that the spread in this type of environment, in NLOS configuration, marked privileged paths of propagation through the radio openings (windows and doors). Other measurement campaigns are planned to characterize the MIMO channel by taking into account the wave polarization. A ray tracing simulation of the MIMO radio channel was presented. It shows that the description of the environments is crucial. Then, a time-efficient implementation of MIMO models, required for link level simulation, was also described. A large number of measurements and simulations will be required to obtain statistical results leading to realistic MIMO channel models for different environments.

\section{Acknowledgements}

This work is part of the "Palmyre" and "Techim@ges" projects with the financial support of Region Bretagne. The authors would like to thank Ronan Cosquer, Julien Guillet, Guy Grunfelder, Alvaro Carcelen and Thierry Tenoux for their contributions to the achievement of sounder, measurement campaigns and their exploitation. They also thank Yannick Chartois and Carlos Pereira for developments related to simulations.

\section{References}

[1] G. J. Foschini, and M. J. Gans, On limits of wireless communications in a fading environment when using multiple antennas, IEEE Wireless Personal Communications, vol. 6, no. 3, (March 1998) 311-335.

[2] D. Gesbert, et al., From theory to practice: An overview of MIMO space-time coded wireless systems, IEEE Journal on Selected Areas in Communications, vol. 21, no. 3, (April 2003) 281-301

[3] P. Guguen, G. El Zein, Les techniques multi-antennes pour les réseaux sans fil, Edition Hermes, Paris, 2004.

[4] K. Yu, and B. Ottersten, Models for MIMO propagation channels, a review, Special Issue on Adaptive Antennas and MIMO Systems, Wiley Journal on Wireless Communications and Mobile Computing, vol. 2, no. 7, (November 2002) 653-666.

[5] M. A. Jensen, and J. W. Wallace, A review of antennas and propagation for MIMO wireless communications, IEEE Transactions on Antennas and Propagation, vol. AP-52, no. 11, (November 2004) 2810-2824.

[6] L. Schumacher, L. T. Berger, and J. Ramiro-Moreno, Recent advances in propagation characterisation and multiple antenna processing in the 3GPP framework, Proceedings of XXVIth URSI General Assembly, Maastricht, The Netherlands, (August 2002).

[7] J. Guillet, Caractérisation et modélisation spatio-temporelles du canal de propagation radioélectrique dans le contexte MIMO, Ph.D. Thesis, INSA Rennes, (July 2004). 
[8] P. Pajusco, Propagation channel models for mobile communication, C. R. Physique 7 (2006) 703-714.

[9] P. Almers, et al., Survey of channel and radio propagation models for wireless MIMO systems, Eurasip journal on Wireless Communications and Networking, Article ID 19070, (2007).

[10] L. M. Correia, Ed., Wireless Flexible Personalised Communications, John Wiley \& Sons, Inc., 2001.

[11] A. F. Molisch, et al., The COST259 directional channel model-I. Overview and methodology, IEEE Transactions on Wireless Communications, vol. 5, no. 12, (December 2006) 3421-3433.

[12] H. Asplund, et al., The COST259 directional channel model-II. Macrocells, IEEE Transactions on Wireless Communications, vol. 5, no. 12, (December 2006) 3434-3450.

[13] N. Czink, et al., Proposal for the COST 273 channel model: how to model multi-cluster environments, COST273, TD-05-070, (January 2005).

[14] L. M. Correia, Mobile Broadband Multimedia Networks, Academic Press, 2006.

[15] N. Czink, and C. Oestges, The COST 273 MIMO channel model - A short tutorial, COST2100, TD-08-405, (February 2008).

[16] www.cost2100.org

[17] P. Kyösti, et al., WINNER II channel models, Technical report WINNER II D1.1.2, V1.2, (September 2007).

[18] M. Narandžić, R. S. Thomä et P. Kyösti, WINNER reference propagation scenarios, COST2100, TD-08-459, (February 2008).

[19] J. P. Kermoal, et al., A stochastic MIMO radio channel model with experimental validation, IEEE Journal on Selected Areas in Communications, vol. 20, no. 6, (August 2002) 1211-1226.

[20] H. Özcelik, et al., Deficiencies of the Kronecker MIMO radio channel model, Electron. Lett., vol. 39, (August 2003) 1209-1210.

[21] W. Weichselberger, Spatial structure of multiple antenna radio channel - a signal processing viewpoint, Ph.D. Thesis, Technischen Universität Wien, Fakultät für Elektrotechnik und Informationstechnik, (December 2003).

[22] M. Liénard, et al., Investigation on MIMO channels in subway tunnels, IEEE Journal on Selected Areas in Communications, vol. 21, no. 3, (April 2003) 332-339.

[23] P. A. Bello, Characterization of randomly time-variant linear channels, IEEE Transactions on Communications, (December 1963) 360-393.

[24] P. C. F. Eggers, Angular-temporal domain analogies of the short-term mobile radio propagation channel at the base station, IEEE Personal, Indoor and Mobile Radio Communications Symposium, (October 1996) 742-746.

[25] G. Durgin, Theory of stochastic local area channel modeling for wireless communications, Phd. Thesis in Electrical Engineering, Faculty of the Virginia Polytechnic Institute and State University, (December 2000).

[26] S. Salous, Multiple input multiple output systems: capacity and channel measurements, Proceedings of the Seventh World Multiconference on Systemics, Cybernetics and Informatics (SCI2003), Florida, USA, (July 2003) 1-5.

[27] M. Steinbauer, A. F. Molisch, E. Bonek, The double-directional radio channel, IEEE Antennas \& Propagation Magazine, vol. 43, no. 4, (2001) $51-63$.

[28] H. Farhat, et al., A Dual Band MIMO channel sounder at 2.2 and $3.5 \mathrm{GHz}$, Proceedings of The IEEE International Instrumentation and Measurement Technology Conference, (I2MTC '08), Victoria, Vancouver Island, Canada (May 2008).

[29] M. Haardt, Efficient one-, two and multidimensional high resolution array signal processing, Ph.D. Thesis, TU-Munchen, (1996).

[30] B. H. Fleury et al., Channel parameter estimation in mobile radio environments using the SAGE algorithm, IEEE Journal on Selected Areas in Communications, vol. 17, no. 3, (March 1999) 434-449.

[31] C. M. Tan et al., On the application of circular arrays in direction finding, Part I: Investigation into the estimation algorithms, Companion paper in 1st Annual COST 273 Workshop, Espoo, Finland, (May 2002).

[32] Y. Lostanlen et al.,Wideband outdoor-to-indoor MIMO channel measurements at $3.5 \mathrm{GHz}$, Proceedings of The $3^{\text {rd }}$ European Conference on Antennas and Propagation, (EuCAP '09), Berlin, Germany, (March 2009).

[33] R. Vauzelle, Y. Pousset, F. Escarieu, A sensitivity study for an indoor channel simulation, Annals of telecommunication, vol. 59, no. 5-6, Annales des Télécommunications, (May 2004) 655-672.

[34] L. Aveneau, P. Combeau, Path finding based on Monte Carlo techniques compared with a full ray-tracing approach in narrow and wide bands, VTC2004, Milan, Italia, (May 2004).

[35] C. Pereira, et al., Sensitivity of the MIMO channel characterization to the modeling of the environment, IEEE Transactions on Antennas and Propagation, vol. 57, no. 4, (2009) 1218-1227.

[36] M. F. Iskander, Z. Yun, Z. Zhang, Outdoor/indoor propagation modeling for wireless communications systems, IEEE AP-S Int. Symp. Dig., USNC/URSI Nat. Radio Sci. Meeting, vol. 2, (July 2001) 150-153.

[37] C. Pereira, Etude avancée des canaux de transmission radio en contexte MIMO: environnements complexes et couplage inter-antennes très large bande, Ph.D. Thesis, Université de Poitiers, (December 2008).

[38] J. M. Conrat, P. Pajusco, A versatile propagation channel simulator for MIMO link level simulation, EURASIP Journal on Wireless Communications and Networking, (2007).

[39] K. H. Ng, E. K. Tameh, A. R. Nix, Modelling and performance prediction for multiple antenna systems using enhanced ray tracing, (2005).

[40] J. Kivinen, Z. Xiongwen, P. Vainikainen, Empirical characterization of wideband indoor radio channel at 5.3 GHz, IEEE Transactions on Antennas and Propagation, vol. 49, (2001) 1192.

[41] Z. Xiongwen, et al., Characterization of Doppler spectra for mobile communications at 5.3 GHz, IEEE Transactions on Vehicular Technology, vol. 52, (2003) 14.

[42] L. Yunxin, H. Xiaojing, The simulation of independent Rayleigh faders, IEEE Transactions on Communications, vol. 50, (2002) 1503.

[43] Z. Yahong Rosa, X. Chengshan, Simulation models with correct statistical properties for Rayleigh fading channels, IEEE Transactions on Communications, vol. 51, (2003) 920.

[44] M. F. Pop, N. C. Beaulieu, Limitations of sum-of-sinusoids fading channel simulators, IEEE Transactions on Communications, vol. 49, (2001) 699.

[45] M. Patzold, F. Laue, Statistical properties of Jakes' fading channel simulator, 48th IEEE Vehicular Technology Conference, (1998).

[46] S. Morosi, et al., Implementation of a wideband directional channel model for a units link level simulator, Globecom, (2003).

[47] M. C. Jeruchim, P. Balaban, K. S. Shanmugan, Simulation of Communication Systems, Plenum Press, New York, 1992.

[48] E. C. Ifeachor, B. W. Jervis, Digital Signal Processing: a Pratical Approach, Addison-Wesley, 1993.

[49] H. Farhat, et al., Mobile radio channels characterization based on wideband SIMO $1 \times 8$ measurements at $2.2 \mathrm{GHz}$, Proceedings of the 5th International Conference on ITS Telecommunications (ITST '05), Brest, (June 2005). 